\title{
TRATAMENTO NÃO OPERATÓRIO DO TRAUMA DE VÍSCERAS ABDOMINAIS PARENQUIMATOSAS
}

\author{
NONOPERATIVE TREATMENT OF THE SOLID ABDOMINAL ORGAN INJURIES
}

Gerson A Pereira Júnior', Júlia Batista de Carvalho², Geraldo S Prado Neto ${ }^{3}$, Juliana R Guedes ${ }^{3}$

\begin{abstract}
${ }^{1}$ Médico Assistente. Unidade de Emergência. Hospital das Clínicas da Faculdade de Medicina de Ribeirão Preto - USP. Docente. Emergências Médicas e Habilidades Cirúrgicas. Curso de Medicina da Universidade de Ribeirão Preto - UNAERP. ${ }^{2}$ Médica. Residente de Clínica Médica. Universidade de Ribeirão Preto - UNAERP. ${ }^{3}$ Doutorandos. Curso de Medicina da Universidade de Ribeirão Preto UNAERP.

CoRResPondêncIA: Dr. Gerson Alves Pereira Júnior. Rua Bernardino de Campos, 1000. Higienópolis. 14030-150 - Ribeirão Preto - SP e-mail: gersonapj@netsite.com.br
\end{abstract}

Pereira Júnior GA, Carvalho JB, Prado Neto GS, Guedes JR. Tratamento não operatório do trauma de vísceras abdominais parenquimatosas. Medicina (Ribeirão Preto) 2007; 40 (4): 538-50, out./dez.

RESUMO: O tratamento não operatório das lesões de órgãos parenquimatosos abdominais (fígado, baço e rins) em pacientes com estabilidade hemodinâmica tem se tornado o método de escolha na última década. A TC é indispensável para a adequada seleção do paciente e para excluir outras lesões que podem necessitar de laparotomia. As estratégias de tratamento não operatório consistem da observação clínica e monitorização cuidadosa com ou sem o uso adjunto da angiografia. A utilização disseminada de embolização angiográfica tem aumentado o número e o tipo de pacientes que podem ser tratados sem cirurgia. O aumento desta modalidade de tratamento não operatório é baseado nas baixas taxas de falha terapêutica relatado na maioria dos estudos. As taxas de sucesso do tratamento não operatório é maior de $90 \%$ para estas lesões. Este artigo de revisão discutirá os conceitos atuais no manuseio não operatório, incluindo o diagnóstico, a seleção dos pacientes, as estratégias utilizadas no tratamento não operatório, os benefícios, os riscos e as complicações.

Descritores: Traumatismos Abdominais. Tratamento não Operatório. Fígado; lesões. Baço; lesões. Rim; lesões.

\section{1- INTRODUÇÃO}

A mudança da rotina de tratamento operatório de escolha, para o tratamento não operatório no trauma abdominal fechado de órgãos sólidos é uma das mais notáveis alterações na abordagem do trauma das últimas duas décadas. ${ }^{1}$

Historicamente, o trauma de baço era tratado por esplenectomia total até a descrição dos primeiros casos de infecções fulminantes na década de 1950. A partir de então, iniciou-se uma tendência na literatura médica advogando o uso de técnicas cirúrgicas conservadoras, particularmente a esplenorrafia, como alternativa à ressecção completa, permitindo a preservação da função esplênica. Com a evolução e maior utilização dos métodos de diagnóstico por imagem, a partir da década de 1980, os pacientes pediátricos com estabilidade hemodinâmica passaram a ser tratados sem cirurgia, com inúmeras vantagens, além da preservação do órgão: menor tempo de internação, menor número de complicações intra-abdominais, menor necessidade de transfusão e menores custos hospi- 
talares. Pouco tempo depois, os mesmos princípios foram utilizados para os pacientes pediátricos com trauma hepático e com traumas combinados de vísceras parenquimatosas abdominais com sucesso similar. ${ }^{2}$

Estes estudos encorajaram os cirurgiões a aplicar a mesma abordagem não operatória para os pacientes adultos.

Em contraste com a alteração de conduta na abordagem do trauma hepático e esplênico, o tratamento recomendado para o trauma renal tem sido não operatório há várias décadas. Diferentemente da hemorragia intraperitoneal do trauma de fígado e baço, a localização retroperitoneal dos rins auxilia no tamponamento da hemorragia em caso de lesão e o rico suprimento sangüíneo pode promover a adequada cicatrização após lesões parenquimatosas. ${ }^{3}$ A conduta conservadora deve ser adotada somente após o adequado estadiamento da lesão. O objetivo de tal conduta é permitir um tratamento seguro ao paciente, evitando-se uma cirurgia desnecessária, muitas vezes, com ressecção parcial ou total do órgão, bem como todos os riscos inerentes ao procedimento cirúrgico e anestésico.

\section{2- TRATAMENTO NÃO OPERATÓRIO}

A conduta conservadora não operatória tem-se tornado o tratamento de escolha em pacientes vítimas de trauma contuso de órgãos parenquimatosos abdominais que apresentam estabilidade hemodinâmica. ${ }^{4}$

O exame tomográfico do abdome tem substituído outros exames de imagem para a avaliação de pacientes hemodinamicamente estáveis. É realizado em pacientes nos quais o abdome não pode ser avaliado adequadamente pelo exame físico devido à alteração do nível de consciência (traumatismo cranioencefálico, etilismo agudo ou abuso de drogas depressoras do sistema nervoso central), naqueles cujos achados no exame físico abdominal não são confiáveis (trauma raquimedular) e naqueles com significante fratura pélvica ou história de múltiplas cirurgias. ${ }^{5}$

O uso da tomografia computadorizada (TC) nos pacientes com trauma abdominal contuso propicia o tratamento conservador não operatório das lesões de órgãos parenquimatosos, diminuindo a necessidade de cirurgia exploradora e reduzindo a freqüência de laparotomias não terapêuticas. A tendência atual em direção ao tratamento conservador não operatório de muitas lesões do fígado, baço e rins é devido, em parte, à capacidade da TC não apenas de definir a presença da lesão e a sua extensão, mas também de excluir outras le- sões significantes, evitando cirurgias desnecessárias. ${ }^{5}$

A decisão final a respeito da indicação de exploração cirúrgica deve ser tomada com base nos achados tomográficos, em conjunção com o quadro clínico completo, principalmente, a necessidade de reposição volêmica para a manutenção da estabilidade hemodinâmica e a experiência de um cirurgião. ${ }^{6}$

Nos traumas abdominais penetrantes, apesar do forte consenso em favor da cirurgia mandatória independentemente dos sinais clínicos apresentados, os ferimentos por arma branca começaram a ter uma abordagem mais seletiva. Nos pacientes que estão relativamente assintomáticos (podendo referir dor no local do ferimento), permitindo a realização de exames físicos seriados, a exploração local do ferimento na parede abdominal anterior, o lavado peritoneal diagnóstico e a TC com triplo contraste em ferimentos do flanco ou dorso, podem permitir o tratamento não operatório. ${ }^{7} \mathrm{O}$ tratamento não operatório utilizado em pacientes selecionados por ferimentos por arma branca podem evitar a laparotomia não terapêutica em 50\% dos pacientes com lesão na parede abdominal anterior e até $85 \%$ daqueles com lesões no flanco ou dorso. ${ }^{3}$

O sucesso da abordagem conservadora não operatório para lesões de órgãos parenquimatosos em casos de trauma abdominal contuso e para casos selecionados de ferimentos por arma branca aceitos na maioria dos grandes centros de trauma, encorajou a conduta conservadora não operatória em pacientes com ferimentos penetrantes por arma de fogo. Apesar do risco de lesão associada em mais de $95 \%$ dos $\operatorname{casos}^{8}$, tal conduta passou a ser adotada no subgrupo de pacientes sem penetração da cavidade peritoneal, que algumas vezes pode ser clinicamente óbvia e, outras vezes, requer a confirmação através de exames de imagem, particularmente, a TC. ${ }^{9}$

Dois argumentos utilizados para justificar o tratamento conservador não operatório para ferimentos penetrantes abdominais, tanto por arma branca, quanto por arma de fogo, tem sido a significante morbidade, os custos associados à laparotomia não terapêuti$\mathrm{ca}^{10}$ e o significante potencial para cicatrização de órgãos parenquimatosos (fígado, baço e rins) traumatizados como observado no tratamento de lesões graves desses órgãos no trauma contuso. ${ }^{11}$

Um estudo recente mostrou que, em casos de ferimentos penetrantes por arma branca ou de fogo, $39,6 \%$ das lesões hepáticas, 30,4\% das lesões renais e $10,7 \%$ das lesões esplênicas podem ser tratadas sem cirurgia, quando não há outras lesões intra-abdominais significantes. ${ }^{3}$ 


\section{1- Critérios de seleção dos pacientes para o tratamento não operatório}

Os critérios utilizados para selecionar os pacientes para a conduta conservadora não operatória incluem a reposição volêmica mínima, ausência de significante traumatismo cranioencefálico, ausência de lesões intra-abdominais concomitantes, idade menor que 55 anos e ausência de outras lesões que possam influenciar a hemorragia ou requerer intervenção cirúrgica. ${ }^{4}$

A presença de lesões múltiplas e a idade avançada permanecem como contra-indicações relativas ao tratamento conservador, embora estudos recentes estejam refutando tais critérios. A experiência obtida em vários estudos realizados com pacientes pediátricos permite tentar tal tratamento em pacientes adultos com múltiplas lesões sem aumento da morbidade e mortalidade comparado com pacientes com lesão isolada de orgão parenquimatoso abdominal. ${ }^{4}$

A necessidade de intervenção cirúrgica para lesões não parenquimatosas tem sido uma contra-indicação relativa ao tratamento conservador, uma vez que tais procedimentos introduzem um potencial para a perda de sangue bem como elimina a possibilidade do seguimento do exame abdominal do paciente enquanto ele estiver anestesiado. ${ }^{4}$

É controverso se a presença de alteração do estado mental devido a traumatismo cranioencefálico permite o tratamento não operatório, pois os resultados de vários trabalhos são conflitantes. ${ }^{12}$

A idade maior que 55 anos tem sido utilizada como contra-indicação para tratamento conservador não operatório para lesões esplênicas. As razões aventadas são a falta de reserva fisiológica nos idosos, bem como as alterações no parênquima do orgão que faz com haja menor probabilidade da hemorragia cessar. ${ }^{13}$

A presença de múltiplas lesões de órgãos parenquimatosos abdominais tem sido uma contra-indicação relativa para o tratamento não operatório. ${ }^{4}$

\section{2- Estadiamento das lesões parenquimatosas abdominais}

O Comitê da "Organ Injury Scaling" (OIS) foi criado em 1987 dentro da Associação Americana de Cirurgia do Trauma para classificar o estadiamento e atualizar periodicamente os graus de lesões dos diversos órgãos. Sua classificação tem sido aceita mundialmente, estabelece cinco graus de lesões em ordem crescente de gravidade. Tal classificação é uti- lizada para o estadiamento das lesões através da realização dos exames radiológicos ou pelo achado intraoperatório no momento da exploração cirúrgica. As Tabelas I, II e III mostram as classificações da OIS para o trauma esplênico, hepático e renal, respectivamente. ${ }^{14,15}$

As lesões de graus 1, 2 e 3 são consideradas "minor", enquanto que as lesões de graus 4 e 5 são consideradas "major". 15

\section{3- Critérios de sucesso e falha no tratamento não operatório}

O tratamento não operatório consiste de observação rigorosa intra-hospitalar, não necessariamente em leito de unidade de terapia intensiva, repouso relativo no leito, dosagens seriadas do hematócrito, hidratação endovenosa abundante, jejum por 24 a 48 horas, ou seja, até uma melhor definição da não necessidade cirúrgica, e antibioticoprofilaxia. ${ }^{16}$

Os preditores de falha do tratamento não operatório são a presença de hipotensão na admissão, o alto grau de lesão, o extravasamento ativo de contraste no CT e a necessidade de transfusão sangüínea. A idade avançada e a lesão neurológica associada, anteriormente citados até como contra-indicação do tratamento não operatório, nos estudos mais recentes parecem não interferir neste tipo de conduta. ${ }^{1}$

Um estudo recente mostrou que na presença de lesão renal ou esplênica com Focused Abdominal Sonography for Trauma (FAST) positivo, uma estimativa de líquido livre no CT maior do que $300 \mathrm{ml}$ e necessidade de hemotransfusão, o risco de falha no tratamento não operatório é de $96 \%$, enquanto que na ausência destes fatores, o risco de falha é de apenas $2 \%{ }^{1}$ A presença destes fatores de risco podem indicar a internação do paciente em terapia intensiva.

Este estudo também mostrou que o tratamento não operatório das lesões esplênicas, particularmente aquelas de grau 3 ou mais, são mais propensas à falhas do que as lesões do rim ou fígado. ${ }^{1}$

A presença de lesões de vísceras ocas em pacientes submetidos ao tratamento não operatório de vísceras parenquimatosas é rara e ocorre em menos de $1 \%$ dos casos. A TC pode mostrar edema da parede intestinal e a observação clínica rigorosa mostra a presença de sinais de irritação peritoneal cada vez mais evidentes. ${ }^{1}$

A seguir, serão discutidos, individualmente, a abordagem dos traumas esplênico, hepático e renal. 
Tabela 1: Estadiamento do trauma esplênico, de acordo com a Organ Injury Scaling (Moore et al. 1995) ${ }^{14}$

\section{Grau Descrição da lesão}

Abreviatted Injury Scale (AIS)

Hematoma (I) Subcapsular, menos de 10\% da superfície acometida.

Laceração

Hematoma (II)

Laceração

Ruptura da cápsula, menor que $1 \mathrm{~cm}$ de profundidade no parênquima.

Subcapsular, acometimento de $10-50 \%$ da superfície;

intraparenquimatoso, menor que $5 \mathrm{~cm}$ de diâmetro.

$1-3 \mathrm{~cm}$ de profundidade no parênquima que não envolve veia trabecular

2

2

2

2

2

Hematoma (III)

Laceração

Subcapsular, maior que 50\% de superfície ou em expansão; ruptura do

3

hematoma subcapsular ou do hematoma parenquimatoso; hematoma

intraparenquimatoso maior que $5 \mathrm{~cm}$ ou em expansão

Maior que $3 \mathrm{~cm}$ de profundidade no parênquima ou envolvendo veias

trabeculares

3

Laceração (IV)

Lacerações envolvendo veias hilares ou segmentares produzindo

desvascularização significativa ( $>25 \%$ do baço)

4

5

Laceração (V)

Vascular

Baço multifragmentado

Avulsão do hilo que desvasculariza o baço.

(

(1)

Escala de lesão esplênica (revisão 1994)

Tabela II: Estadiamento do trauma hepático, de acordo com a Organ Injury Scaling (Moore et al. 1995) ${ }^{14}$

\section{Grau Descrição da lesão}

Hematoma (I)
Laceração

Hematoma (II) Subcapsular, 10-50\% de área; intraparenquimatoso, menor que $10 \mathrm{~cm} \mathrm{de}$

Laceração

Hematoma (III)
Laceração

$1-3 \mathrm{~cm}$ de profundidade no parênquima, menor que $10 \mathrm{~cm}$ de extensão

Subcapsular, envolvendo menos de $10 \%$ da superfície

Laceração da cápsula, menor que $1 \mathrm{~cm}$ de profundidade no parênquima

Abreviatted Injury

Scale (AIS)

Subcapsular, maior que 50\% de superfície de área ou em expansão; ruptura do hematoma subcapsular ou do hematoma parenquimatoso Maior que $3 \mathrm{~cm}$ de profundidade

2

2

2

2

3

3

Laceração (IV) Ruptura do parênquima envolvendo 25-75\% de um lobo hepático ou 1 a 3 segmentos de Couinaud dentro de um lobo isolado

Laceração (V) Ruptura do parênquima envolvendo mais que $75 \%$ do lobo hepático ou

Vascular

mais que 3 segmentos de Couinaud dentro de um lobo isolado

Lesão venosa justahepática; p.e. veia cava retrohepática / veias hepáticas principais

2

(1)

(1)

(1)

(VI) Vascular Avulsão hepática

6 


\section{3- TRAUMA ESPLÊNICO}

As lesões do baço ocorrem mais freqüentemente associadas ao trauma contuso em acidentes automobilísticos, quedas e agressões físicas. Podem ocorrer de forma isolada ou associadas à outras lesões intra-abdominais.

Em um estudo com grande casuística, as lesões penetrantes foram tratadas sem cirurgia em $62,4 \%$ dos casos e nos traumas contusos em $85,6 \%$ dos casos. A taxa de tratamento não operatório em pacientes com mais de 18 anos de idade com lesões esplênicas foi de $81,8 \%$, enquanto em menores de 18 anos de idade foi de $91,8 \%{ }^{2}$.

A Figura 1 mostra os vários graus de estadiamento das lesões esplênicas na TC de abdome.

Pacientes submetidos à cirurgia antes de 12 horas da admissão hospitalar geralmente são considerados como tratamento cirúrgico, enquanto que paci- entes submetidos a cirurgia depois de 12 horas ou mais da admissão, são considerados como falha do tratamento conservador. ${ }^{2}$ Os benefícios do tratamento conservador devem ser pesados em relação aos riscos de deixar de diagnosticar outras lesões e no atraso do tratamento.

O tratamento conservador no trauma esplênico consiste na simples observação clínica ou angiografia, com ou sem embolização. Assim, podemos ter diferentes estratégias no tratamento conservador: ${ }^{17}$

- observação: o período de observação de um paciente com trauma esplênico deve ser feito em ambiente monitorizado ou de cuidados intensivos. O paciente deve ser mantido em jejum e com avaliações seriados do hematócrito.

- angiografia: a angiografia é uma terapia adjuvante nas lesões esplênicas que tem sido extensivamente investigada. O primeiro relato do uso da angiografia
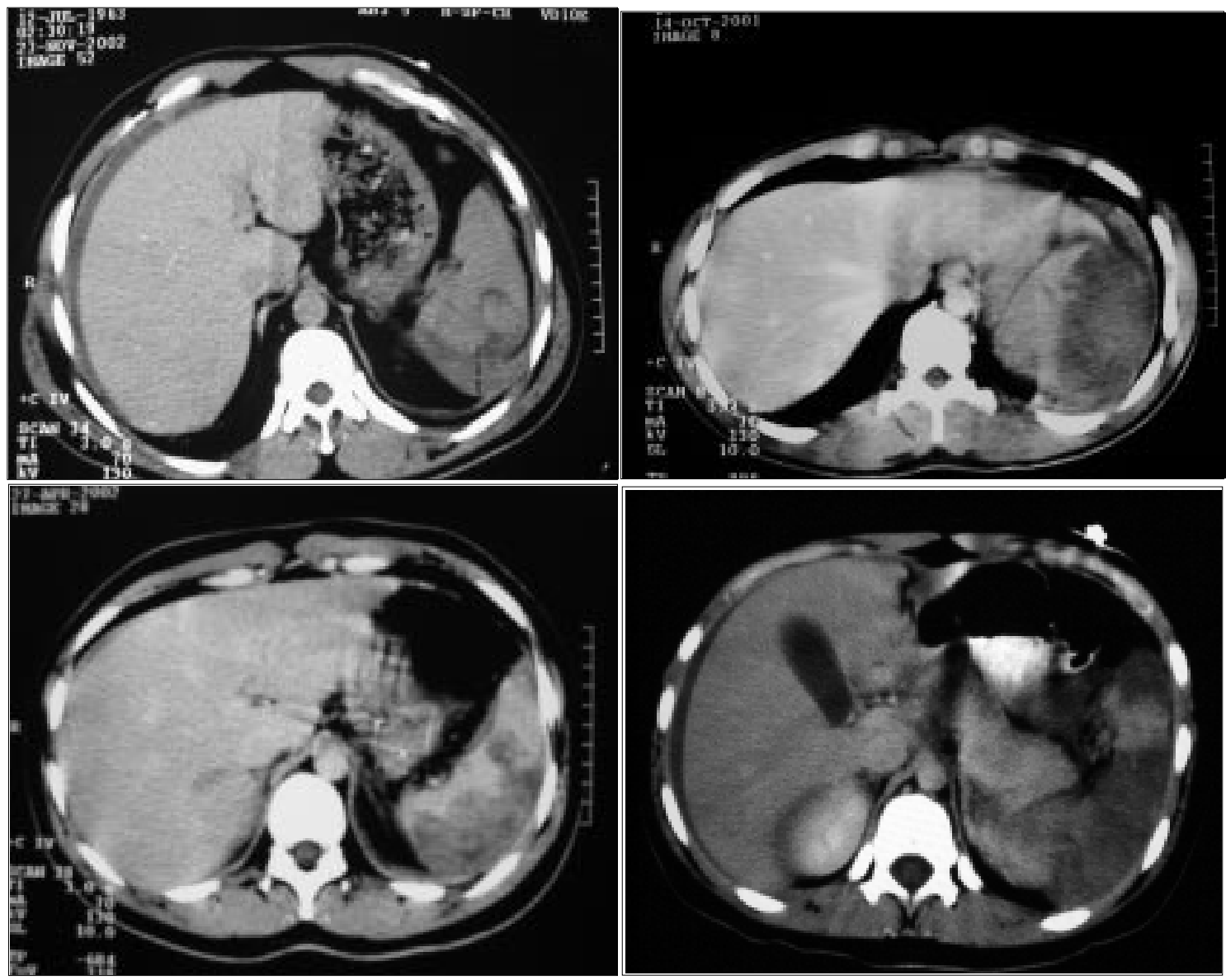

Figura 1: Exemplos de trauma esplênico. A) Lesão grau II - Laceração do parênquima menor que $3 \mathrm{~cm}$. B) Lesão grau III - hematoma subcapsular > 50\%. C) Lesão grau IV - desvascularização $>25 \%$ do parênquima. D) Lesão grau V - baço multifragmentado. 
com embolização seletiva como terapia adjuvante na lesão esplênica foi feita em 1995. Taxas de sucesso de $97 \%$ foram demonstradas para embolização de artéria proximal neste estudo. Estudos mais recentes, têm sugerido angiografia seletiva para pacientes com lesões de alto grau ou com extravasamento de contraste no CT. Outra indicação para angiografia inclui persistência de taquicardia e queda do hematócrito. ${ }^{17} \mathrm{Na}$ técnica de angiografia, pode ser utilizada a embolização proximal ou a embolização seletiva. A primeira não controla diretamente o sangramento, mas diminui a pressão no baço permitindo a hemostasia intrínseca, enquanto a perfusão esplênica é mantida por via colateral. $\mathrm{O}$ segundo tipo permite a hemostasia direta das áreas lesadas. Estas técnicas também podem ser combinadas.

A taxa de complicações de uma laparotomia não terapêutica pode chegar a $15 \%$. As complicações de uma esplenectomia, incluem: um aumento do risco de infecções e enfraquecimento do sistema imune secundário à asplenia.. ${ }^{17}$

A única contra-indicação absoluta do tratamento não operatório é o paciente com instabilidade hemodinâmica que não responde à reposição de fluidos. ${ }^{17}$ Outras contra-indicações que são debatidas na literatura são: altos graus de lesão no CT, extravasamento ativo de contraste no CT, FAST positivo, hemoperitônio volumoso, ISS $>25$ e necessidade de transfusão de sangue. ${ }^{15}$

Estudos mais recentes sugerem que os pacientes com lesão neurológica associada podem ser tratados conservadoramente com sucesso, inclusive havendo uma possibilidade de melhor prognóstico, por evitar a morbidade da anestesia e da laparotomia. ${ }^{17}$

A idade maior que 55 anos tem sido uma contra-indicação histórica para tratamento conservador. Os estudos são conflitantes, porém dados mais recentes demonstraram que a idade isoladamente não é um fator que contra-indica o tratamento conservador. ${ }^{17}$

O seguimento com TC de abdome, geralmente, não é feito em lesões de baixo grau. Geralmente, a repetição do exame é feita de acordo com a sintomatologia clínica ou em lesões de grau avançado após 24 a 72 horas.

As falhas do tratamento não operatório podem resultar de hemorragias significativas e, subseqüente, hipotensão e choque circulatório. As falhas geralmente são corrigidas com esplenectomia, ao invés de se tentar uma esplenorrafia. Estas falhas no tratamento conservador indicam a necessidade de intervenção cirúrgica. As taxas de falha são bastante variáveis.
Uma revisão recente demonstrou taxa de sucesso de 98\% em crianças e $83 \%$ em adultos com lesões de grau I a III. ${ }^{17}$

Uma revisão retrospectiva foi publicada em 2000 com análise de 1488 pacientes com lesão esplênica. Trinta e oito por cento foram manejadas com cirurgia. $\mathrm{O}$ restante foi manejado conservadoramente. A taxa de falha no tratamento conservador foi de $10,8 \%$. De acordo com o grau da lesão, as taxas de falhas foram de $4,8 \%$ no grau I, $9,5 \%$ no grau II, $16,9 \%$ no grau III, $33,3 \%$ no grau IV e $75 \%$ no grau $\mathrm{V}^{18}$.

Os fatores de risco para falha terapêutica têm sido exaustivamente estudados. Em diversos estudos a taxa de falha no tratamento conservador foi associada ao grau de hemoperitôneo e o grau da lesão. O extravasamento de contraste no CT também pode predizer a taxa de falha do tratamento somente com observação, indicando a necessidade de angiografia. O FAST positivo e a necessidade de transfusão sanguínea, são fatores de risco adicionais na falha do tratamento conservador.

As complicações do tratamento não operatório, além da hemorragia, inclui o risco de lesões associadas desapercebidas. Quando se utilizam técnicas angiográficas, além destas complicações, podemos ter a nefropatia induzida pelo contraste, o abscesso e o infarto esplênico.

\section{4- TRAUMA HEPÁTICO}

O mecanismo de trauma mais frequente é o contuso devido a acidentes de transporte, seguido de agressões físicas. ${ }^{2}$

A Figura 2 mostra os vários graus de estadiamento das lesões hepáticas na TC de abdome.

$\mathrm{Na}$ maioria dos estudos, a taxa de tratamento não operatório do trauma hepático durante o período do estudo variou de 74,6 a $87,1 \%$. $^{2}$

O taxa de tratamento não operatório foi maior nos seguintes casos: presença de mecanismo contuso de lesão e AIS mais baixo. Em contraste, a falha do tratamento não operatório foi maior quando associado a aumento da pressão sistólica no atendimento inicial e a idade do paciente. ${ }^{2}$

No tratamento não operatório, o paciente pode ser submetido à simples observação ou, angiografia com ou sem embolização. A observação deve ser feita em um ambiente monitorizado, com exame abdominal seriado e hematócrito. Pacientes com lesões de grau maior em tratamento conservador estão sob maior risco de falha do tratamento. ${ }^{17}$ 

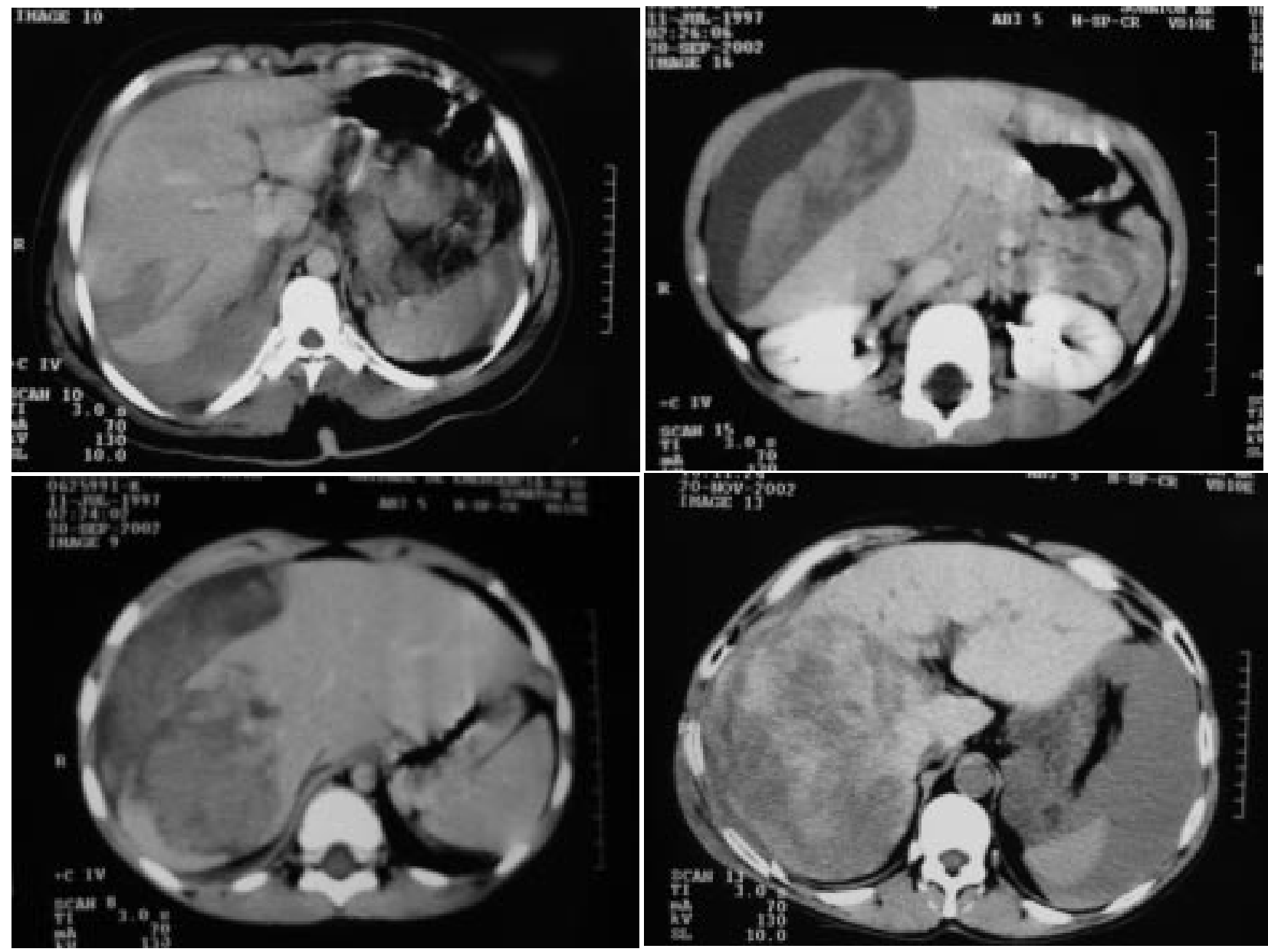

Figura 2: Exemplos de trauma hepático. A) Lesão grau II - Laceração do parênquima menor que $3 \mathrm{~cm}$. B) Lesão grau III - hematoma subcapsular $>50 \%$. C) Lesão grau IV - ruptura do parênquima envolvendo $25-75 \%$ do lobo direito. D) Lesão grau V - ruptura do parênquima envolvendo mais de $75 \%$ do lobo direito, com hemoperitônio volumoso.

A embolização por angiografia é uma técnica bastante útil no tratamento não operatório. Em vários estudos, as lesões de grau mais avançado, IV e V foram manejadas com sucesso somente com embolização. O uso rápido da embolização pode ser benéfico. ${ }^{15}$

Além do beneficio óbvio de evitar a morbidade de uma laparotomia, o tratamento conservador das lesões hepáticas tem demonstrado outros benefícios, incluindo menor necessidade de transfusão sangüínea, menor índice de complicações abdominais, menor tempo de permanência hospitalar e menor tempo de permanência em unidades de terapia intensiva. ${ }^{17}$

Não existe um consenso para o seguimento com TC de abdome. Geralmente não é feito em lesões de baixo grau e a repetição do exame é realizada de acordo com a sintomatologia clínica ou em lesões de grau avançado após 7 a 10 horas. ${ }^{6,17}$
Os fatores de risco para falhas do tratamento não operatório têm variado em diferentes estudos. $\mathrm{O}$ maior grau da lesão, pooling de contraste na tomografia, o tracking periportal de sangue, volume do hemoperitônio e a presença de múltiplas lesões de órgãos parenquimatosos. No entanto, vários trabalhos recentes, principalmente utilizando técnicas angiográficas divergem em relação aos fatores de risco de falha do tratamento não operatório. ${ }^{2}$

As complicações associadas ao tratamento não operatório do trauma hepático diferem daquelas atribuídas ao trauma esplênico devido às peculiaridades anatômicas e fisiológicas do fígado. Assim, podemos ter abscessos, fístulas biliares, biliomas e hemobilia. ${ }^{6,17}$

Os abscessos e biliomas podem ser tratados com elevada taxa de sucesso por meio da drenagem percutânea. As lesões biliares podem ser tratadas com a utilização da drenagem biliar por meio da colangio- 
grafia endoscópica retrógrada. A hemobilia pode ser tratada com técnicas angiográficas. ${ }^{4,17}$

Uma outra complicação atribuída ao tratamento das lesões hepáticas de grau elevado é o desenvolvimento da síndrome de compartimento abdominal. ${ }^{17}$

Os estudos diferem quanto à frequiência destas complicações com variações de 5 a $12 \%$. As taxas mais relatadas nas várias publicações são: hemorragias $(5 \%)$, abscessos $(0,7 \%)$, lesões desapercebidas $(0,5 \%)$ e biliomas $(0,4 \%) ., 4,6,17$

\section{5- TRAUMA RENAL}

A maioria dos casos de trauma renal é devido ao trauma contuso ( 85 a 90\%) e o restante devido aos ferimentos penetrantes. Porém, tais porcentagens sobre o mecanismo de trauma são variáveis, de acordo com a população e a região geográfica estudada. ${ }^{19}$

A maioria das lesões renais ocorre como resultado de um trauma direto ou da rápida desaceleração causada por acidentes de tráfego, quedas, lesões esportivas ou agressões físicas. ${ }^{16}$

Atualmente, as indicações para o estudo radiológico no trauma renal estão melhor estabelecidas na literatura médica mundial. Tais indicações foram definidas a partir de extensos trabalhos realizados por diversos autores em diferentes partes do mundo, o que representou uma grande economia em termos de tempo de avaliação e custos do tratamento total.

As indicações para a investigação radiológica na suspeita de trauma renal são as seguintes:

1- presença de hematúria macroscópica na avaliação inicial, independente do estado hemodinâmico do paciente. ${ }^{20}$

2- hematúria microscópica no paciente que chega à sala de admissão com choque circulatório (Pressão arterial sistólica menor ou igual a $90 \mathrm{mmHg}$ ).

3- trauma com grande desaceleração, o que deve ser suspeitado devido ao mecanismo de trauma, como queda de altura e trauma direto em região dorsal e, devido as lesões associadas como fratura vertebral toraco-lombar, fratura das 3 últimas costelas inferiores e fratura/deslocamento do processo transverso de L1 e L2. ${ }^{21}$

4- ferimento penetrante nas proximidades dos rins. ${ }^{22}$

Os pacientes politraumatizados que estão normotensos e sem hematúria macroscópica não necessitam de exame para a detecção de hematúria microscópica e não há indicação de investigação radiológica. $^{22}$
A hematúria microscópica isoladamente é um pobre indicador de lesão renal significante. ${ }^{21}$

Está definido que o grau de hematúria não se correlaciona com o tipo e a extensão da lesão renal e cerca de $25 \%$ dos casos de lesão da artéria renal não apresentam hematúria. ${ }^{23}$

Além da presença de hematúria macroscópica, devemos prestar atenção em traumas cujo mecanismo sugere impacto com grande desaceleração, tais como certos tipos de colisões automobilísticas e quedas de altura. ${ }^{24}$

A TC suplantou a urografia excretora como o exame de imagem de escolha para a avaliação de pacientes politraumatizados com suspeita de trauma renal na maioria dos grandes centros de trauma. ${ }^{24}$

Numerosos estudos mostraram as limitações da urografia excretora para estadiamento das lesões renais e comprovaram a superioridade da TC na definição dos detalhes anatômicos, mensuração do tamanho do hematoma perirenal e da profundidade da laceração renal, presença de extravasamento urinário, integridade dos vasos renais e a presença e extensão das lesões extra-renais. ${ }^{24}$

A TC mostrou ser capaz de fazer uma adequada identificação das lesões do pedículo renal, sendo a arteriografia indicada apenas quando os resultados da tomografia não são conclusivos. ${ }^{25}$

Não estando a TC disponível para avaliação radiológica, a urografia excretora é um método aceitável, embora a sua acurácia para estadiamento de pacientes com trauma renal varie de 68 a $95 \% .^{26}$

A utilização da ultrassonografia na avaliação do trauma renal permite a identificação de líquido livre na cavidade peritoneal, sem a preocupação de fazer o diagnóstico topográfico do órgão lesado. Pode fazer a avaliação do contorno e arquitetura do parênquima renal e definir a presença de hematoma ou outras lesões retroperitoneais. Entre as principais desvantagens da sua utilização pode-se destacar o fato de ser um exame onde a experiência e o treinamento individual para sua realização são extremamente importantes (operador dependente), a não distinção entre sangue e urina e a difícil avaliação do pedículo renal. ${ }^{27} \mathrm{~A}$ tecnologia atualmente disponível não permite um adequado estadiamento, porém o aperfeiçoamento tecnológico poderá aumentar a participação desta modalidade diagnóstica. É excelente para o seguimento de lesões renais já estadiadas e submetidas ao tratamento conservador ou cirúrgico para diagnóstico de complicações precoces, principalmente as alterações no tamanho dos 
hematomas intra-renal, subcapsular, perirenal ou retroperitoneal, bem como do extravasamento urinário. ${ }^{27}$

A classificação da Organ Injury Scaling (OIS) estabelece o sistema de gravidade de lesão demonstrado na Tabela III. A Figura 3 mostra os vários graus de estadiamento das lesões renais na TC de abdome.

As lesões de grau 1 a 3 são consideradas "minor" e as lesões de grau 4 e 5 são classificadas como "major". 15

Na maioria dos estudos, cerca de $85 \%$ destas lesões são classificadas como "minor", correspondendo aos graus de 1 a 3 pela OIS e são tratadas por meio de observação. Cerca de 5\% dos casos refere-se a lesões do pedículo ou rim multifragmentado, classificadas como grau 5. Dependendo das condições clínicas dos pacientes, estas lesões freqüentemente requerem intervenção cirúrgica imediata. ${ }^{24}$ Os $10 \%$ remanescentes dos traumas renais contusos são lacerações "major", sendo classificados como grau 4. O tratamento destas lesões permanece controverso, com opiniões divididas entre aqueles a favor da abordagem cirúrgica imediata contra àqueles a favor da conduta expectante. ${ }^{16}$

O tratamento conservador não operatório consiste de observação rigorosa intra-hospitalar, não necessariamente em leito de unidade de terapia intensi- va, repouso relativo no leito, dosagens seriadas do hematócrito, hidratação endovenosa abundante, jejum por 24 a 48 horas, ou seja, até uma melhor definição da não necessidade cirúrgica e antibioticoprofilaxia. ${ }^{16}$

O uso profilático de antibióticos é questionável quanto à sua indicação e qual o tipo de medicamento a ser usado. ${ }^{16}$

Os pacientes recebem alta hospitalar assim que cessar a hematúria macroscópica, sendo orientados a manter o repouso domiciliar por, pelo menos 2 semanas, com retorno gradual às atividades até que, por volta de 6 semanas após a resolução da hematúria macroscópica, retorne às atividades de rotina. ${ }^{16}$

O tratamento das lesões renais de grau 1, 2 e 3, consideradas "minor" não é controverso, uma vez que a totalidade dos casos é submetida ao tratamento conservador não operatório, com raras complicações. ${ }^{16}$

A tendência atual no tratamento do trauma renal grau 4 é retardar a cirurgia, mesmo em casos de extravazamento de urina ou presença de tecido renal desvitalizado, exceto se houver lesões associadas, principalmente de pâncreas e cólon, na TC. ${ }^{28}$ No entanto, o manuseio conservador de lesões renais com fragmentos desvitalizados resulta em maior morbidade urológica (38\%) e um risco associado de necessidade de nefrectomia tardia de $6 \%{ }^{28}$

Tabela III: Estadiamento do trauma renal, de acordo com a Organ Injury Scaling (Scaling (Moore et al. 1989) ${ }^{15}$

\begin{tabular}{|c|c|c|}
\hline Grau & Descrição da lesão & $\begin{array}{l}\text { Abreviatted Injury } \\
\quad \text { Scale (AIS) }\end{array}$ \\
\hline $\begin{array}{l}\text { Contusão } \\
\text { (I) }\end{array}$ & $\begin{array}{l}\text { Hematúria macro ou microscópica, com estudo radiológico urológico } \\
\text { normal. }\end{array}$ & 1 \\
\hline Hematoma & Subcapsular, não expansível sem laceração do parênquima. & 1 \\
\hline $\begin{array}{l}\text { Hematoma } \\
\text { (II) }\end{array}$ & $\begin{array}{l}\text { Hematoma peri-renal não expansível confinado à loja renal retroperito- } \\
\text { neal. }\end{array}$ & 2 \\
\hline Laceração & $\begin{array}{l}1 \mathrm{~cm} \text { de profundidade do cortex renal, sem ruptura do sistema coletor } \\
\text { ou extravazamento de urina. }\end{array}$ & 2 \\
\hline $\begin{array}{c}\text { (III) } \\
\text { Laceração }\end{array}$ & $\begin{array}{l}\text { Maior que } 1 \mathrm{~cm} \text { de profundidade no parênquima renal, sem ruptura } \\
\text { calicial ou extravazamento urinário. }\end{array}$ & 3 \\
\hline $\begin{array}{l}\text { Laceração } \\
\quad(\mathbf{I V})\end{array}$ & $\begin{array}{l}\text { Laceração do parênquima estendendo-se através do cortex renal, } \\
\text { medula e sistema coletor. }\end{array}$ & 4 \\
\hline Vascular & Lesão da artéria ou veia renal principal com hemorragia contida. & 4 \\
\hline $\begin{array}{c}\text { Laceração } \\
\text { (V) } \\
\text { Vascular }\end{array}$ & $\begin{array}{l}\text { Rim completamente fraturado. } \\
\text { Avulsão do hilo renal que desvasculariza o rim. }\end{array}$ & $\begin{array}{l}5 \\
5\end{array}$ \\
\hline
\end{tabular}


A
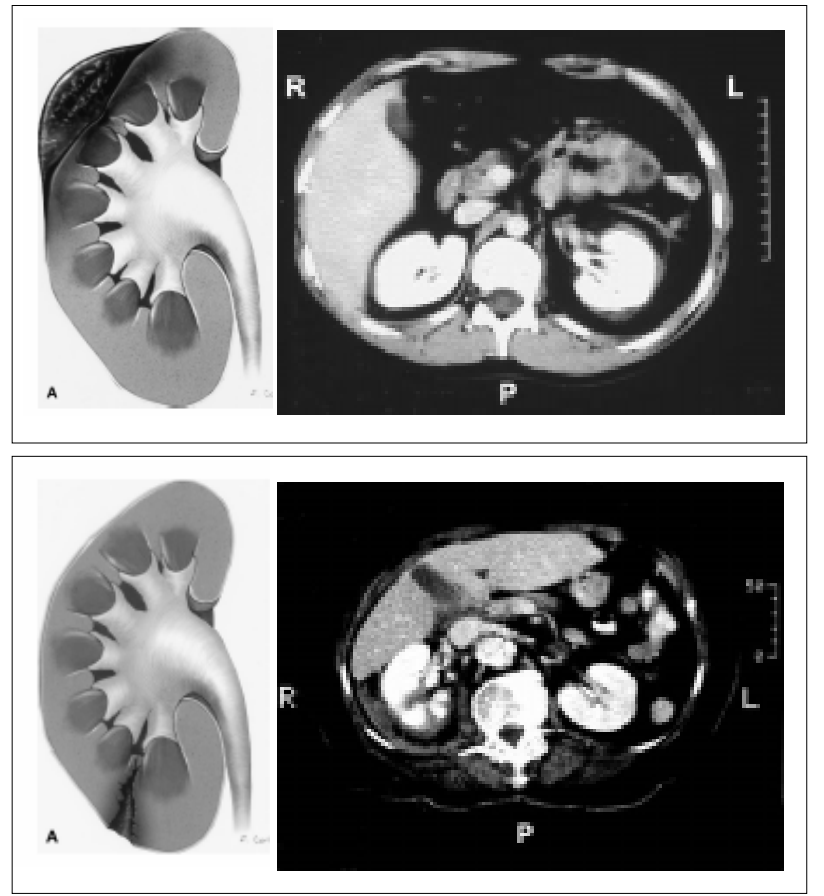

C

$\mathbf{E}$

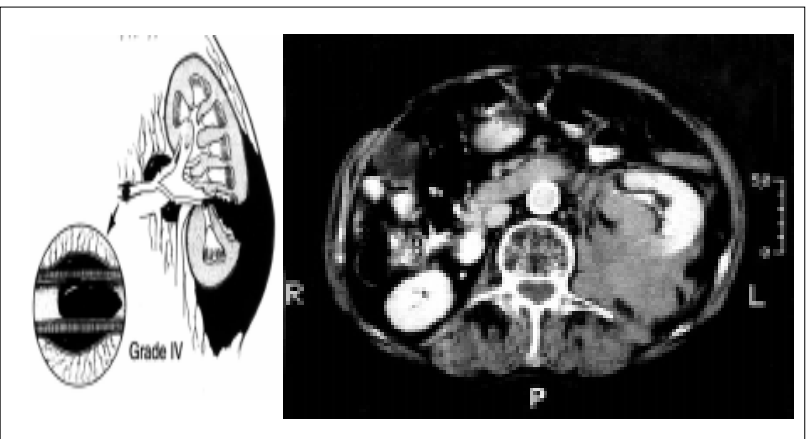

B
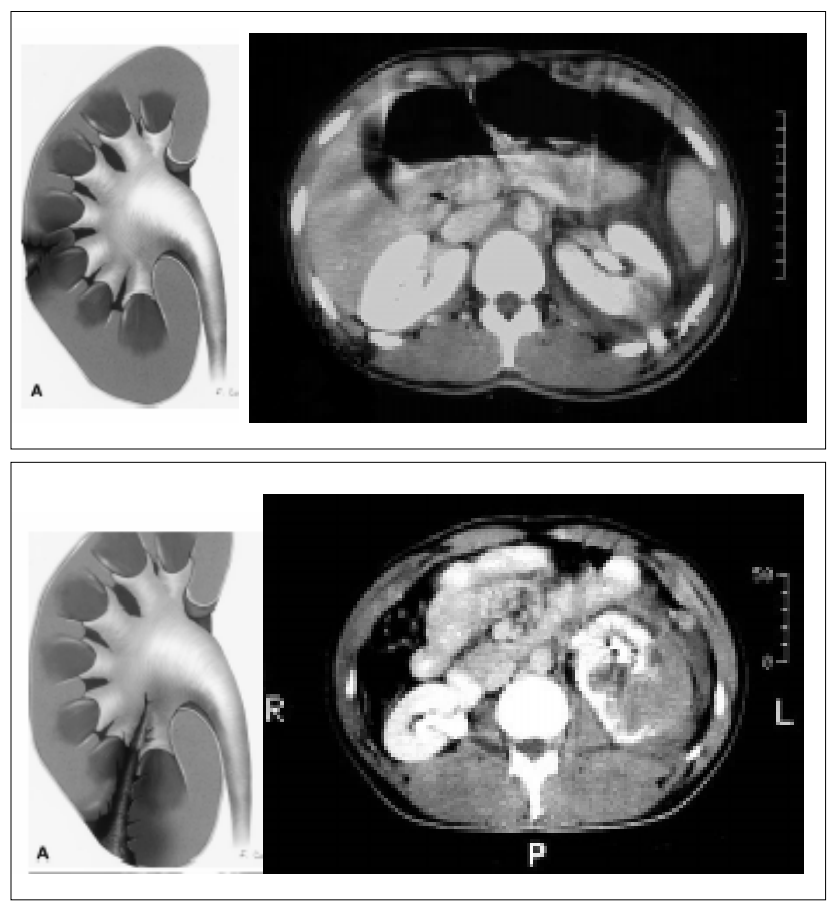

D

F

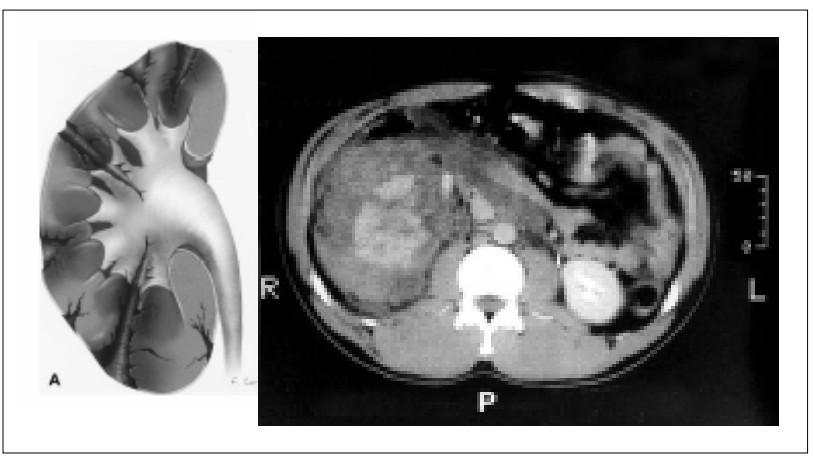

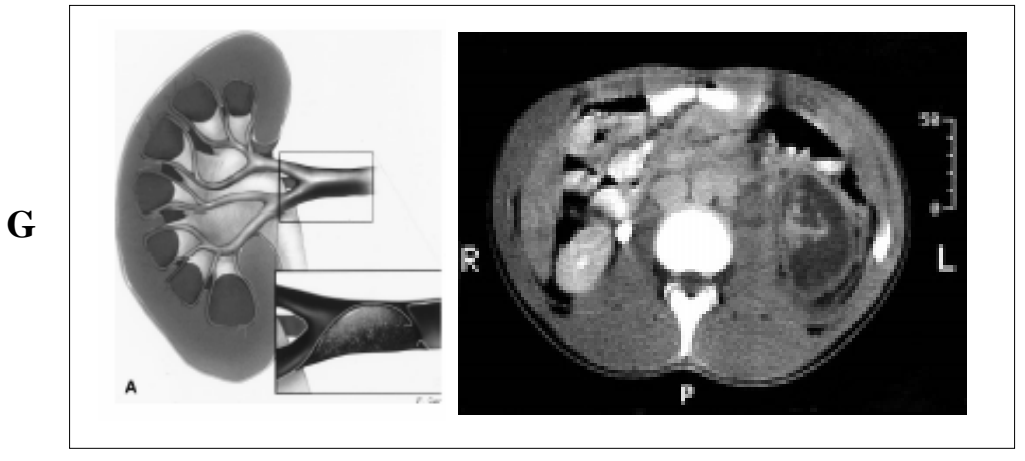

Figura 3: Exemplos de trauma renal com representação esquemática das lesões. A) Lesão grau I - hematoma subcapsular do rim esquerdo, B) Lesão grau II - laceração do parênquima renal esquerdo menor que $1 \mathrm{~cm}, \mathrm{C}$ ) Lesão grau III - laceração do parênquima renal direito maior que $1 \mathrm{~cm}$, sem extravasamento urinário, D) Lesão grau IV - laceração do parênquima renal esquerdo com extravasamento urinário, E) Lesão grau IV - ruptura do pedículo vascular esquerdo contido à loja renal, F) Lesão grau $\mathrm{V}$ - rim direito multifragmentado e G) Lesão grau V - desvascularização total de rim esquerdo, com contrastação apenas da cápsula renal - sinal do rim cortical. 
Não é o extravasamento de urina por si que dita o tratamento, mas a gravidade da lesão renal subjacente e se a pelve renal e o ureter estão drenando urina adequadamente.

O tratamento dos casos de trombose da artéria renal permanece controverso, com alguns autores advogando a tentativa de reparo cirúrgico precoce e outros apenas a observação dos casos, o que resultará na perda de função do órgão na grande maioria dos pacientes. A tendência é considerar a correção cirúrgica apenas em pacientes hemodinamicamente estáveis com rins solitários ou lesões bilaterais das artérias renais. $\mathrm{O}$ tratamento conservador não operatório nas lesões renovasculares tem uma taxa de sucesso que varia de 39 a $63 \% .^{16}$

As lesões renais grau 5, do tipo de múltiplas lacerações renais, não são frequentes e os estudos apresentam um número pequeno de pacientes. No entanto, parece que o tratamento conservador não operatório é viável em casos selecionados ou em pacientes jovens sem comorbidades e que apresentam estabilidade hemodinâmica. ${ }^{24}$

Contrastando com a baixa necessidade de exploração cirúrgica do trauma renal contuso, historicamente, há uma elevada indicação cirúrgica em pacientes com trauma renal penetrante. As indicações absolutas para exploração incluem a identificação de hematoma perirenal pulsátil e em expansão, hemorragia persistente de uma lesão renal conhecida com queda dos valores hematimétricos e a lesão dos vasos do pedículo renal. As indicações relativas para exploração renal incluem o extravasamento urinário óbvio, presença de um rim multifragmentado, presença de tecido desvitalizado e a incapacidade do estadiamento adequado da lesão renal. ${ }^{28}$

O tratamento das lesões renais penetrantes está lentamente mudando da exploração cirúrgica imediata para uma conduta não operatória em casos selecionados. Esta alteração é primariamente devido ao estabelecimento de protocolos de diagnóstico clínico, laboratorial e radiológico capazes de uma acurada definição anatômica da lesão permitindo o estadiamento seguro da sua gravidade. ${ }^{29}$

O tratamento conservador não cirúrgico de traumas penetrantes tem sido mais comumente adotado em casos de ferimentos por arma branca, quando há menos lesões intra-abdominais associadas e menor necessidade transfusional. ${ }^{28}$

Como regra geral, em casos de trauma penetrante, a decisão intra-operatória deve ser tomada avaliando-se os sinais de estabilidade hemodinâmica, grau de lesão renal e a presença de lesões associadas. Todos os pacientes com instabilidade hemodinâmica apresentando lesões intra-abdominais devem ser explorados. Os pacientes que apresentam estabilidade hemodinâmica com lesões renais grau 1 ou 2 podem ser tratados apenas com observação. A forte associação de sangramento tardio em lesões de graus maiores (grau 3 ou 4) demandam exploração renal sincrônica para pacientes submetidos a laparotomia para lesões não renais. ${ }^{29}$

As lesões renais mais significativas são mais freqüentes em pacientes com traumatismos multissistêmicos. A maioria das lesões renais isoladas é de pequena gravidade, podendo ser tratadas conservadoramente. Outras lesões associadas com trauma renal seguindo trauma contuso multissistêmico incluem: fraturas de extremidades, lesões torácicas, fratura pélvica, lesões intra-abdominais, traumatismo craniencefálico e ruptura do diafragma. O fígado e o baço são os órgãos abdominais mais comumente lesados associados ao trauma renal, seguidos pelo pâncreas, cólon e intestino delgado. ${ }^{2}$

As complicações do tratamento conservador não operatório são: urinoma, abscesso perinefrético, hemorragia tardia e a hipertensão arterial. ${ }^{2}$

Um extravasamento urinário persistente ou a formação de um urinoma perirenal é a complicação mais freqüente do tratamento conservador não operatório citada na literatura. ${ }^{16}$

O principal erro que se pode cometer nestes graus de lesão é o estadiamento inadequado, o que implica geralmente em complicações como urinoma e sangramento persistente. ${ }^{30}$

A presença de urinoma não é indicação absoluta de cirurgia. No caso de urinomas de pequeno volume, geralmente a resolução ocorre espontaneamente e, assim, adota-se uma conduta expectante com seguimento ultrassonográfico até sua total absorção. $\mathrm{Na}$ presença de urinomas de grande volume ou de pequeno volume com aumento progressivo, pode ser tentada a drenagem percutânea guiada pela ultrassom ou, mais apropriadamente pela TC. ${ }^{31}$

Pode-se, ainda, realizar uma cistoscopia e proceder a colocação de cateter de "pig-tail" para minimizar o extravasamento de urina. ${ }^{31}$

Os riscos tardios do tratamento conservador do urinoma são a fibrose peri-renal e peri-ureteral, obstrução pelvi-ureteral e infundibular, infecção e hipertensão arterial. $^{31}$ 
No caso de abscesso perinefrético, assim como no urinoma infectado, faz-se a abordagem cirúrgica por via extraperitoneal com evacuação e drenagem local apropriada. ${ }^{31}$

A hemorragia pode ocorrer de forma precoce ou tardia durante a fase de recuperação dos pacientes. A hemorragia pode ser perinefrética ou urinária recorrente e manifesta-se com alteração do quadro hemodinâmico ou queda progressiva do hematócrito, dependendo da intensidade e rapidez do sangramento. Seu tratamento poderá variar desde conservador com hidratação, hemotransfusão e repouso, até embolização por arteriografia ou cirurgia. ${ }^{31}$
A hipertensão arterial no seguimento tardio dos pacientes com trauma renal permanece como uma grande controvérsia na literatura, com resultados conflitantes entre os diversos trabalhos de diferentes autores, com sua incidência no tratamento não operatório variando de 6 a 55\% e, no tratamento cirúrgico variando de zero a $29 \%$. $^{31}$

Os dois mecanismos considerados responsáveis pela hipertensão pós-traumática são uma resposta mediada pela renina ao infarto renal total ou segmentar e a compressão do parênquima renal devido a um hematoma subcapsular que, subseqüentemente, organiza numa cápsula de fibrina ("Page kidney).16

Pereira Júnior GA, Carvalho JB, Prado Neto GS, Guedes JR. Nonoperative treatment of the solid abdominal organ injuries. Medicina (Ribeirão Preto) 2007; 40 (4): 538-50, oct./dec.

ABSTRACT: Nonoperative management of the solid organ injuries (liver, spleen and kidneys) in hemodynamically patients has become the standard of care in the last decade. Computed tomography scan is invaluable in determining appropriate patient selection and to exclude other injuries that may necessitate laparotomy. Nonoperative management strategies primarily consist of careful observation with or without the use of adjunctive angiography. The widespread use of angiographic embolization has increased the number and type of patients that can be safety managed without operative intervention. The increasing use of nonoperative management is based on the low failure rates reported in most studies. Success rates of nonoperative treatment have increase to $>90 \%$ for these injuries. Practitioners must remain vigilant, however, because failures of nonoperative management may need immediate intervention. This review will discuss current concepts in nonoperative management, including diagnosis, patient selection, nonoperative treatment strategies, benefits, risks, and complications.

Keywords: Abdominal Injuries. Nonoperative Management. Liver; injuries. Spleen; injuries; Kidney; injuries.

\section{REFERENCIAS}

1 - Velmahos GC, Toutouzas KG, Radin R, Chan L, Demetriades D. Nonoperative treatment of blunt injury to solid abdominal organs. Arch Surg 2003, 138: 844-51.

2 - Hurtuk M, Reed RL, Esposito TJ, Davis KA, Luchette FA. Trauma surgeons practice what they preach: the NTDB story on solid organ injury management. J Trauma 2006, 61:243-55.

3 - Demetriades D, Hadjizacharia P, Constantinou C, Brown C Inaba K, Rhee P, Salim A. Selective nonoperative management of penetrating abdominal solid organ injuries. Ann Surg 2006, 244: $620-8$.

4 - Sartorelli KH, Frumiento C, Rogers FB, Osler TM. Nonoperative management of hepatic, splenic, and renal injuries in adults with multiple injuries. J Trauma 2000; 49: 56-62.
5 - Wolfman NT, Bechtold RE, Scharling ES, Meredith JW. Blunt upper abdominal trauma: evaluation by CT. Am J Roentgenol 1992; 158: 493-501.

6 - Brick SH, Taylor GA, Potter BM, Eichelberger MR. Hepatic and splenic injury in children: role of CT in the decision for laparotomy. Radiology 1987; 165: 643-6.

7 - American College of Surgeons. ACS. Comittee on Trauma. Advanced Trauma Life Support. Instructor manual. 6th ed, Chicago; 1997.

8 - Feliciano DV, Burch JM, Spjut-Patrianely V, Mattox KL, Jordan GL Jr. Abdominal gunshot wounds. An urban trauma center's experience with 300 consecutive patients. Ann Surg 1988; 208: 362-70.

9 - Saadia R, Degiannis E. Non-operative treatment of abdominal gunshot injuries. Br J Surg 2000; 87: 392-7. 
10 - Renz BM, Feliciano DV. The length of hospital stay after unnecessary laparotomy for trauma: a prospective study. $\mathrm{J}$ Trauma 1996; 40: 187-90.

11 - Moudouni SM, Patard JJ, Manunta A, Guiraud P, Guille F, Lobel B. A conservative approach to major blunt renal lacerations with urinary extravasation and devitalized renal segments. BJU Int 2001; 87: 290-4.

12 - Gaunt WT, McCarthy MC, Lambert CS, Anderson GL, Barney LM, Dunn MM et al. Traditional criteria for observation of splenic trauma should be challenged. Am Surg 1999; 65: 689-91.

13 - Barone JE, Burns G, Svehlak SA, Tucker JB,Bell T, Korwin S et al. Management of blunt splenic trauma in patients older than 55 years. Southern Connecticut Regional Trauma Quality Assurance Committee. J Trauma 1999; 46: 87-90.

14 - Moore EE; Cogbill TH; Jurkovich GJ; Shackford SR; Malangoni MA; Champion HR. Organ injury scaling: spleen and liver (1994 revision). J Trauma 1995; 38:323-4.

15 - Moore EE, Shackford SR, Pachter HL, McAninch JW, Browner $B D$, Champion HR et al. Organ injury scaling: spleen, liver and kidney. J Trauma 1989; 29: 1664-6.

16 - Mattheus LA, Spirnak JP. The nonoperative approach to major blunt renal trauma. Sem Urol 1995; 23: 77-82.

17 - Stein D, Scalea TM. Nonoperative management of spleen and liver injuries. J Intensive Care Med 2006, 21:296-303

18 - Peitzman AB, Heil B, Rivera L. Blunt splenic injury in adults: multi institutional study of the Eastern Association for the Surgery of Trauma. J Trauma 2000, 29:177-89.

19 - Cheng DLW, Lazan D,Stone N. Conservative treatment of type III renal trauma. J Trauma 1994; 36: 491-4

20 - Knudson MM, McAninch JW, Gomez R, Lee P, Stubbs HA. Hematuria as a predictor of abdominal injury after blunt trauma. Am J Surg 1992; 164: 482-6.

21 - McAndrew JD, Corriere JN. Radiographic evaluation of renal trauma. Evaluation of 1103 consecutive patients. $\mathrm{Br} \mathrm{J} \mathrm{Urol}$ 1994; 73: 352-4.

22 - Eastham JA, Wilson TG., Ahlering TE. Urological evaluation and management of renal proximity stab wounds. J Urol 1993; 150: 1771-3.
23 - McAninch JW. Injuries to the genitourinary tract. In Tanagho EA, McAninch JW, editors: General urology. 13th edit. Norwalk, Conn: Appleton \& Lange; c1992. Cap. 18, p. 30826.

24 - Altman AL, Haas C, Dinchman KH, Spirnak JP. Selective nonoperative management of blunt grade 5 renal injury. $J$ Urol 2000; 164: 27-31.

25 - Lupetin AR, Mainwaring $\mathrm{BL}$, Daffner $\mathrm{RH}$. CT diagnosis of renal artery injury caused by blunt abdominal trauma. Am J Roentgenol 1989; 153: 1065-8.

26 - Nicolaisen GS, McAninch JW, Marshall GA, Bluth RF Jr, Carroll PR. Renal trauma: re-evaluation of the indications for radiographic assessment. J Urol 1985; 133: 183-6.

27 - Rosales A, Arango O, Coronado J, Vesa J, Maristany J, Gelabert A. The use of ultrasonography as the initial diagnostic exploration in blunt renal trauma. Urol Int 1992; 48: 134-7.

28 - Wessells H, McAninch JW, Meyer A, Bruce J. Criteria for nonoperative treatment of significant penetrating renal injuries. J Urol 1997; 157: 24-7.

29 - Armenakas NA, Duckett CP, McAninch JW. Indications for nonoperative management of renal stab wounds. J Urol 1999; 161: 768-71.

30 - Husmann DA, Morris JS. Attempted nonoperative management of blunt renal lacerations extending through the corticomedullary junction: the short-term and long-term sequelae. J Urol 1990; 143: 682-4.

31 - Husmann DA, Gilling PJ, Perry MO, Morris JS, Boone TB. Major renal lacerations with a desvitalized fragment following blunt abdominal trauma: a comparison between nonoperative (expectant) versus surgical management. J Urol 1993; 150: 1774-7.

Recebido em: 29/06/2007

Aprovado em: 28/08/2007 